Discussion Paper No. 538

ASYMPTOTIC PROPERTIES OF

THE ESTIMATOR OF THE LONG-RUN

COEFFICIENT IN A DYNAMIC MODEL WITH

INTEGRATED REGRESSORS AND

SERIALLY CORRELATED ERRORS

\author{
Koichi Maekawa \\ Michael McAleer \\ and \\ Zonglu He
}

May 2001

The Institute of Social and Economic Research

Osaka University

6-1 Mihogaoka, Ibaraki, Osaka 567-0047, Japan 


\title{
Asymptotic Properties of the Estimator of the Long-run Coefficient in a Dynamic Model with Integrated Regressors and Serially Correlated Errors*
}

\author{
Koichi Maekawa'), Michael McAleer ${ }^{2)}$, and Zonglu $\mathrm{He}^{3)}$ \\ ${ }^{1)}$ Hiroshima University, ${ }^{2)}$ University of Western Australia, ${ }^{3)}$ Aki Women's University \\ Revised: April, 2001
}

\begin{abstract}
In this paper we examine the asymptotic properties of the estimator of the long-run coefficient (LRC) in a dynamic regression model with integrated regressors and serially correlated errors. We show that the OLS estimators of the regression coefficients are inconsistent but the OLS-based estimator of the LRC is superconsistent. Furthermore, we propose an alternative consistent estimator of the LRC, compare the two estimators through a Monte Carlo experiment, and find that the proposed estimator is MSE-superior to the OLS-based estimator.

JEL Classification numbers: C13, C15, C22
\end{abstract}

${ }^{*}$ The authors are grateful to Kimio Morimune, Adrian Pagan, Katsuto Tanaka, Taku Yamamoto, seminar participants at Hitotsubashi University and University of Western Australia for helpful comments on an earlier version of this paper. The first author wishes to thank the Department of Economics at the University of Western Australia for its hospitality during a visit in 1997, and the second author wishes to acknowledge the financial support of the Australian Research Council abd the Institute of Social and Economic Research at Osaka University. This paper was revised while the second author was a Visiting Scholar from Abroad at the Institute. 


\section{Introduction}

An autoregressive distributed lag model with serially correlated disturbances represents an important class of dynamic regression model in econometrics. Such a model containing lagged dependent and lagged independent variables with lag-orders $p$ and $q$, respectively, is called an autoregressive distributed lag model (henceforth, denoted by $A D L(p, q)$ ). An $A D L$ model can be written as

$$
y_{t}=c+A(L) y_{t}+B(L) z_{t}+u_{t}
$$

where $A(L)$ and $B(L)$ are the polynomials of the lag operator $L$ defined by

$$
\begin{aligned}
& A(L)=\alpha_{1} L+\cdots+\alpha_{p} L^{p}, \\
& B(L)=\beta_{0}+\beta_{1} L+\cdots+\beta_{q} L^{q} .
\end{aligned}
$$

The long-run effect of $z$ on $y$ is given by the long-run coefficient, defined as

$$
\delta=\frac{B(1)}{1-A(1)}=\frac{\beta_{0}+\beta_{1}+\cdots+\beta_{q}}{1-\alpha_{1}-\cdots-\alpha_{p}} .
$$

If (1) is regarded as a consumption function with consumption $y$ and income $z, \delta$ is the long-run marginal propensity to consume (abbreviated as $L R M P C)$.

Since many economic time series are nonstationary processes such as integrated or cointegrated processes, we need to develop the asymptotic theory for nonstationary $A D L(p, q)$ models. Furthermore, such models with serially correlated disturbances are important in both theory and practice.

Recently Maekawa, Yamamoto, Takeuchi and Hatanaka (1996, abbreviated as MYTH) dealt with the $A D L(1,0)$ model with an integrated regressor and serially correlated disturbances: namely, $y_{t}=\alpha y_{t-1}+\beta z_{t}+u_{t}$, where $z_{t}$ is integrated of order 1 . When $u_{t}$ is assumed to be a stationary $A R(1)$ process, MYTH showed that $\hat{\alpha}$ and $\beta$ are $\sqrt{T}$-inconsistent and asymptotically normally distributed (see Corollary 3 below).

He, Maekawa, and McAleer (1998) (henceforth HMM) dealt with a similar model, $y_{t}=c+\alpha y_{t-1}+\beta z_{t}+u_{t}$, and analysed a non-linear estimator $\hat{\delta}=\hat{\beta} /(1-\hat{\alpha})$ for the $L R M P C$ in this model, that is, $\delta=\beta /(1-\alpha)$, where $\hat{\alpha}$ and $\hat{\beta}$ are $O L S$ estimators. Their model is a special case of a regression model with cointegrated regressors, as examined in Park and Phillips 
(1989, Section 5.2, p.116). However, HMM extended the Park and Phillips model and derived the long-run implications of the estimated coefficients, as well as the asymptotic distribution of the nonlinear estimator $\hat{\delta}$. Park and Phillips did not deal with a nonlinear estimator such as $\hat{\delta}$. As $\hat{\alpha}$ and $\hat{\beta}$ are $\sqrt{T}$-inconsistent when $u_{t}$ is serially correlated, it might be anticipated that $\hat{\delta}$ is also inconsistent. Somewhat strikingly, HMM showed that $\hat{\delta}$ is superconsistent.

This paper extends HMM to a general $A D L(p, q)$ model and proceeds as follows. Section 2 presents the model and the assumptions. Section 3 derives the asymptotic distributions of the $O L S$ estimators of the regression coefficients and the long-run coefficient. Section 4 investigates the asymptotic properties of an alternative estimator of the long-run coefficient. Section 5 compares the small sample distributional properties of two estimators of the long-run coefficient by performing Monte Carlo experiments for the most simple case of the model. Section 6 summarizes the main results of the paper and provides some concluding comments. Detailed derivations and proofs are given in the Appendices to this paper and are available from the authors upon request, and can be downloaded from the web site http://home.hiroshimau.ac.jp/maekawa/index.html.

\section{ADL (p,q) Model}

First we specify the model (1) as follows:

$$
\begin{aligned}
& y_{t}=c+A(L) y_{t}+B(L) z_{t}+u_{t}, \\
& u_{t}=C^{*}(L) u_{t}+v_{t}, \\
& z_{t}=D^{*}(L) z_{t}+\varepsilon_{t}, \quad t=1,2, \cdots, T,
\end{aligned}
$$

where $v_{t} \sim$ i.i.d.N $\left(0, \sigma_{1}^{2}\right), \varepsilon_{t} \sim$ i.i.d.N $\left(0, \sigma_{2}^{2}\right), v_{t}$ and $\varepsilon_{t}$ are independent, and

$$
\begin{aligned}
C^{*}(L) & =c_{1}^{*} L+c_{2}^{*} L^{2}+\cdots+c_{l}^{*} L^{l}, \\
D^{*}(L) & =d_{1}^{*} L+d_{2}^{*} L^{2}+\cdots+d_{s}^{*} L^{s} .
\end{aligned}
$$

Rewrite $u_{t}$ as an infinite moving average process as

$$
u_{t}=C(L) v_{t} \text { with } C(L) \equiv \frac{1}{1-C^{*}(L)}=\sum_{i=1}^{\infty} c_{i} L^{i},
$$


and make the following assumptions:

$A 1$. All the roots of the characteristic equations $1-A(x)=0$ and $1-$ $C^{*}(x)=0$ lie outside the unit circle.

A2. $1-D^{*}(L)=0$ has a single unit root and all the other roots lie outside the unit circle, that is, $1-D^{*}(L)=(1-L) D_{1}(L)$, where all the roots of $D_{1}(L)=0$ lie outside the unit circle.

Defining $D(L) \equiv \frac{1}{(1-L) D_{1}(L)}$, we can rewrite $z_{t}$ as

$$
z_{t}=\frac{\varepsilon_{t}}{1-D^{*}(L)}=\frac{\varepsilon_{t}}{(1-L) D_{1}(L)}=D(L) \varepsilon_{t} .
$$

Using the infinite moving average process, we can rewrite model (5) as

$$
\begin{aligned}
y_{t} & =c+A(L) y_{t}+B(L) z_{t}+u_{t}, \\
u_{t} & =C(L) v_{t}, \\
z_{t} & =D(L) \varepsilon_{t}, \\
t & =1,2, \cdots, T .
\end{aligned}
$$

Note that we can further rewrite model (8) as

$$
y_{t-1}=\tilde{c}+\delta(L) z_{t-1}+\phi(L) v_{t-1},
$$

where

$$
\begin{aligned}
\delta(L) & =\frac{B(L)}{1-A(L)}=\sum_{i=0}^{\infty} \delta_{i} L^{i}, \\
\phi(L) & =\frac{C(L)}{1-A(L)}=\sum_{i=0}^{\infty} \phi_{i} L^{i}, \\
\tilde{c} & =\frac{c}{1-A(1)} .
\end{aligned}
$$

From assumptions $A 1$ and $A 2$, we have the following lemma: 


\section{Lemma 1}

$$
\begin{gathered}
\text { (i) } \sum_{i=0}^{\infty}\left|\phi_{i}\right|<\infty, \\
\text { (ii) } \sum_{i=0}^{\infty} i\left|\delta_{i}\right|<\infty, \\
\text { (iii) } \sum_{i=0}^{\infty}\left|c_{i}\right|<\infty \\
\text { (iv) } \sum_{i=0}^{\infty}\left|d_{i}\right|<\infty .
\end{gathered}
$$

where $d_{i}$ is defined by $\frac{1}{D_{1}(L)}=\sum_{i=1}^{\infty} d_{i} L^{i}=d(L)$.

$\mathbf{P}$ roof. ilable on request, as an extension of HMM.

\section{Asymptotic Distributions of $\hat{\alpha}, \hat{\beta}, \hat{c}$ and $\hat{\delta}$}

In this section we investigate the asymptotic properties of $\hat{c}, \hat{\alpha}$, and $\hat{\beta}$. Equation (9) can be rewritten as

$$
y_{t-1}=\tilde{c}+\delta z_{t}+a_{t}
$$

where $a_{t}$ is expressed as an infinite moving average of $v_{t}$ and $\varepsilon_{t}$ as follows:

$$
a_{t}=\sum_{i=0}^{\infty} \sum_{j=0}^{\infty} \gamma_{i}^{*} d_{j} \varepsilon_{t-i-j}+\sum_{i=0}^{\infty} \phi_{i} v_{t-i-1}
$$

where $\gamma_{0}^{*}=-\delta, \gamma_{i}^{*}=-\left(\delta_{i}+\delta_{i+1}+\cdots\right), i=1,2, \cdots$.

This formula is useful for obtaining the asymptotic distributions below. Note that Equation (12) suggests that $y_{t-1}$ and $z_{t}$ are cointegrated, so that our model has cointegrated regressors. This could be called "stochastic multicollinearity", which yields the asymptotic normality of $\hat{c}, \hat{\alpha}$ and $\hat{\beta}$, in spite of the presence of integrated variables in the model. 
To derive the asymptotic distributions of $\hat{c}, \hat{\alpha}$ and $\hat{\beta}$, we introduce the Brownian motions $B_{1}$ and $B_{2}$, defined by

$$
\frac{1}{\sqrt{T}} \sum_{t=1}^{[T r]} v_{t} \Rightarrow B_{1}(r), \frac{1}{\sqrt{T}} \sum_{t=1}^{[T r]} \varepsilon_{t} \Rightarrow B_{2}(r), r \in[0,1],
$$

where $\Rightarrow$ signifies weak convergence and $[x]$ denotes the integer part of $x$. Following the algebra of non-standard asymptotics in Phillips (1987), we obtain the following results:

Theorem $2 \quad(\sqrt{T}-$ inconsistency of $\hat{c}, \hat{\alpha}$ and $\hat{\beta})$ Under Assumptions $A 1$ and $A 2$, we have:

(a) $\sqrt{T}\left(\hat{\alpha}_{i}-\alpha_{i}-\alpha_{i}^{*}\right) \Rightarrow N\left(0, \sigma_{\alpha_{i}}^{2}\right), i=1,2, \cdots, p$,

(b) $\sqrt{T}\left(\hat{\beta}_{j}-\beta_{j}-\beta_{j}^{*}\right) \Rightarrow N\left(0, \sigma_{\beta_{j}}^{2}\right), j=0,1,2, \cdots, q$,

(c) $\sqrt{T}\left(\hat{c}-c-c^{*}\right) \Rightarrow-\tilde{c} N\left(0, \sigma_{c}^{2}\right)+f\left(B_{1}, B_{2}\right)$,

(d) $\delta \sqrt{T}\left(\sum_{i=1}^{p} \hat{\alpha}_{i}-\sum_{i=1}^{p} \alpha_{i}\right)+\sqrt{T}\left(\sum_{i=0}^{q} \hat{\beta}_{i}-\sum_{i=0}^{q} \beta_{i}\right) \stackrel{p}{\rightarrow} 0$

where $\alpha_{i}^{*}$ and $\beta_{j}^{*}$ are some constants comprising the coefficient $c_{i}$ for $i=$ $1,2, \cdots$ in the lag polynomial $C(L)$ in (8), and vanish when all $c_{i}$ are zeros or there is no serial correlation in $u_{t}$. On the other hand, $\sigma_{\alpha_{i}}^{2}$ and $\sigma_{\beta_{j}}^{2}$ are the asymptotic variances and $f\left(B_{1}, B_{2}\right)$ is a functional of the Brownian motion $B_{1}(r)$ and $B_{2}(r)$. The precise expressions for these constants and function are obtained as extensions of HMM and given in the Appendix to the paper.

$\mathbf{P}$ roof. Available on request as Section $\mathrm{C}$ in the Appendix to the paper.

Remark 1 We note from (a), (b) and (c) that $\hat{c}, \hat{\alpha}$ and $\hat{\beta}$ are $\sqrt{T}$-consistent only if $u_{t}$ is not serially correlated. From (d), we note that the asymptotic distribution of $\delta \sqrt{T}\left(\sum_{i=1}^{p} \hat{\alpha}_{i}-\sum_{i=1}^{p} \alpha_{i}\right)+\sqrt{T}\left(\sum_{i=0}^{q} \hat{\beta}_{i}-\sum_{i=0}^{q} \beta_{i}\right)$ degenerates as $T$ approaches infinity. The asymptotic normality arises from the "stochastic multicollinearity", as noted above. 
HMM examined how the serial correlation in $u_{t}$ affects the asymptotic properties of $\hat{\alpha}, \hat{\beta}, \hat{c}$ and $\hat{\delta}$ in the simplest case of $A D L(1,0)$, i.e.,

$$
\begin{gathered}
y_{t}=c+\alpha y_{t-1}+\beta z_{t}+u_{t},|\alpha|<1 \\
u_{t}=\rho u_{t-1}+v_{t},|\rho|<1 \\
z_{t}=z_{t-1}+\varepsilon_{t}, \\
t=1,2, \cdots, T,
\end{gathered}
$$

where $v_{t} \sim i . i . d . N\left(0, \sigma_{1}^{2}\right)$ and $\varepsilon_{t} \sim i . i . d . N\left(0, \sigma_{2}^{2}\right)$ are assumed to be independent. In this case, the asymptotic distributions of $\hat{c}, \hat{\alpha}$ and $\hat{\beta}$ are given as follows:

Corollary 3 In the simplest case (13), we have
(a) $\operatorname{plim}(\hat{\alpha}-\alpha)=\alpha^{*}$;
(b) $\operatorname{plim}(\hat{\beta}-\beta)=\beta^{*}$;
(c) $\operatorname{plim}(\hat{c}-c)=c^{*}$;
(d) $\sqrt{T}\left(\hat{\alpha}-\alpha-\alpha^{*}\right) \Rightarrow N\left(0, \tilde{\sigma}_{1}^{2}\right)$;
(e) $\sqrt{T}\left(\hat{\beta}-\beta-\beta^{*}\right) \Rightarrow \frac{\beta}{1-\alpha} N\left(0, \tilde{\sigma}_{1}^{2}\right)$;
$(f) \sqrt{T}\left(\hat{c}-c-c^{*}\right) \Rightarrow N\left(0, \tilde{\sigma}_{2}^{2}\right)+\frac{1}{1-\rho} \frac{B_{1}(1) \int B_{2}^{2}(r) d r-\int B_{2}(r) d r\left[\int B_{2}(r) d B_{1}(r)-(1-\rho) \gamma^{*} Q_{2}\right]}{\int B_{2}^{2}(r) d r-\left[\int B_{2}(r) d r\right]^{2}}$,

in which

$\gamma^{*}=\frac{P}{Q_{3}}, \delta^{*}=-\frac{\beta}{1-\alpha} \gamma^{*}, c^{*}=-\frac{c}{1-\alpha} \gamma^{*}$,

$\tilde{\sigma}_{1}^{2}=\lambda_{1}^{2} \sigma_{1}^{2} \sigma_{2}^{2}+\lambda_{2}^{2} \sigma_{1}^{4}$,

$\lambda_{1}=-\frac{\beta}{(1-\alpha)^{2}(1-\rho) Q_{3}}, \lambda_{2}=\left[\frac{1}{(1-\alpha)(1-\rho)^{2}}-\frac{\rho}{(1-\alpha \rho)\left(1-\rho^{2}\right)}\right] \frac{1}{Q_{3}}$,

$\tilde{\sigma}_{2}^{2}=\mu_{1}^{2} \sigma_{1}^{2} \sigma_{2}^{2}+\mu_{2}^{2} \sigma_{1}^{4}+\mu_{3}^{2} \sigma_{1}^{2}$,

$\mu_{1}=-\frac{c}{1-\alpha} \lambda_{1}, \mu_{2}=-\frac{c}{1-\alpha} \lambda_{2}, \mu_{3}=\left(\frac{c}{1-\alpha}\right)^{2} \frac{1}{(1-\rho) Q_{3}}$,

$P=\frac{\rho \sigma_{1}^{2}}{(1-\alpha \rho)\left(1-\rho^{2}\right)}$,

$Q_{2}=\left(\frac{1}{1-\alpha}\right)\left(\frac{1}{1-\rho}\right) \int B_{2}(r) d B_{1}(r)-\frac{1}{2}\left(\frac{\beta}{1-\alpha}\right)\left(\frac{1}{1-\alpha}\right)\left[B_{2}^{2}(1)+\sigma_{2}^{2}\right]$,

$Q_{3}=\left(\frac{1}{1-\alpha^{2}}\right)\left(\frac{\beta}{1-\alpha}\right)^{2} \sigma_{2}^{2}+\frac{(1+\alpha \rho) \sigma_{1}^{2}}{\left(1-\alpha^{2}\right)(1-\alpha \rho)\left(1-\rho^{2}\right)}$.

$\mathbf{P}$ roof. lable on request, as an extension of HMM.

Remark 2 Note that $\hat{c}, \hat{\alpha}$ and $\hat{\beta}$ are $\sqrt{T}$-consistent only if there is no serial correlation in $u_{t}$, or $\rho=0$ in model (13). 
Now consider the $O L S$-based estimator,

$$
\hat{\delta}=\frac{\hat{B}(1)}{1-\hat{A}(1)}=\frac{\sum_{j=0}^{q} \hat{\beta}_{j}}{1-\sum_{i=1}^{p} \hat{\alpha}_{i}},
$$

which can be rewritten as

$$
\sqrt{T}(\hat{\delta}-\delta)=\frac{\delta \sqrt{T}\left(\sum_{i=1}^{p} \hat{\alpha}_{i}-\sum_{i=1}^{p} \alpha_{i}\right)+\sqrt{T}\left(\sum_{j=0}^{q} \hat{\beta}_{j}-\sum_{j=0}^{q} \beta_{j}\right)}{1-\sum_{i=1}^{p} \hat{\alpha}_{i}} .
$$

We cannot use $\sqrt{T}$ as the normalizer as the denominator degenerates to obtain the asymptotic distribution of $\hat{\delta}-\delta$ as $T \rightarrow \infty$, as shown in Theorem $2(d)$. Instead, we normalize $(\hat{\delta}-\delta)$ by $T$ to calculate the asymptotic distribution of $\hat{\delta}$ directly as

$$
T(\hat{\delta}-\delta)=\frac{\delta T\left(\sum_{i=1}^{p} \hat{\alpha}_{i}-\sum_{i=1}^{p} \alpha_{i}\right)+T\left(\sum_{j=0}^{q} \hat{\beta}_{j}-\sum_{j=0}^{q} \beta_{j}\right)}{1-\sum_{i=1}^{p} \hat{\alpha}_{i}} .
$$

Theorem 4 In model (5), the asymptotic distribution of $T(\hat{\delta}-\delta)$ is given by

$$
T(\hat{\delta}-\delta) \Rightarrow \frac{f_{a}}{f_{b}}
$$

where

$$
\begin{aligned}
& f_{a}=C(1) d(1)\left[\int B_{2}(r) d B_{1}(r)-B_{1}(1) \int B_{2}(r) d r\right] \\
& -\left[-d(1) \int B_{2}(r) d r\left[\begin{array}{c}
\phi(1) B_{1}(1)- \\
(\delta+\gamma(1)) d(1) B_{2}(1)
\end{array}\right] I_{p}^{\prime}+f_{W Z}^{\prime}\right] A^{11} P_{w u}
\end{aligned}
$$




$$
\begin{aligned}
- & {\left[-d(1)^{2} \int B_{2}(r) d r B_{2}(1) I_{q}^{\prime}+f_{\epsilon Z}^{\prime}\right] A^{21} P_{w u} } \\
f_{b} & =d(1)^{2}\left(1-\sum_{i=1}^{p} \alpha_{i}-\sum_{i=1}^{p} \alpha_{i}^{*}\right)\left\{\int B_{2}^{2}(r) d r-\left[\int B_{2}(r) d r\right]^{2}\right\}
\end{aligned}
$$

where $A^{11}$ and $A^{21}$ are matrices of non-random elements, and $P_{w u}, f_{W Z}$, and $f_{\epsilon Z}$ are matrices of functionals of the Brownian motions $B_{1}$ and $B_{2} ; I_{k}^{\prime}=$ $(1,1 \cdots 1)_{1 \times k}, k=p, q$. The precise formulae are omitted, but are given as extensions of HMM.

$\mathbf{P}$ roof. Available on request, as an extension of HMM.

Remark 3 From Theorems 2 and 4, the asymptotic distributions of $\sqrt{T}\left(\hat{\alpha}_{i}-\right.$ $\left.\alpha_{i}-\alpha_{i}^{*}\right)$ and $\sqrt{T}\left(\hat{\beta}_{j}-\beta_{j}-\beta_{j}^{*}\right)$ are normal, but the asymptotic distributions of $\sqrt{T}\left(\hat{c}-c-c^{*}\right)$ and $T(\hat{\delta}-\delta)$ are non-standard. Furthermore, although the OLS estimators $\hat{c}, \hat{\alpha}_{i}$ and $\hat{\beta}_{j}$ in our model are $\sqrt{T}$-inconsistent, the OLS-based estimator $\hat{\delta}$ is T-consistent, or superconsistent.

Corollary 5 In model (13), the asymptotic distribution of $\hat{\delta}$ collapses to

$$
\begin{aligned}
& T(\hat{\delta}-\delta) \Rightarrow \\
& \frac{\frac{1}{1-\rho} \int B_{2}(r) d B_{1}(r)-\left\{\frac{1}{1-\rho} B_{1}(1)+\frac{\beta}{1-\alpha} \mu B_{2}(1)\right\} \int B_{2}(r) d r+\frac{1}{2} \frac{\beta}{1-\alpha} \mu\left\{B_{2}^{2}(1)+\sigma_{2}^{2}\right\}}{(1-\alpha)\left\{\int B_{2}^{2}(r) d r-\left(\int B_{2}(r) d r\right)^{2}\right\}}
\end{aligned}
$$

where $\mu=\frac{(1+\alpha) \rho \sigma_{1}^{2}}{(1-\alpha \rho)\left(1-\rho^{2}\right)\left(\frac{\beta}{1-\alpha}\right)^{2} \sigma_{2}^{2}+(1-\rho) \sigma_{1}^{2}}$.

\section{$\mathbf{P}$ roof. See HMM.}

As HMM showed that $\int B_{2}(r) d r$ occurs only if $c \neq 0$, that is, when there is a constant term, we have the following:

Corollary 6 When the constant term $c=0$ in model (13), we have 


$$
T(\hat{\delta}-\delta) \Rightarrow \frac{\frac{1}{1-\rho} \int B_{2}(r) d B_{1}(r)+\frac{1}{2} \frac{\beta}{1-\alpha} \mu\left\{B_{2}^{2}(1)+\sigma_{2}^{2}\right\}}{(1-\alpha) \int B_{2}^{2}(r) d r}
$$

Remark 4 Comparing (16) and (17), the asymptotic distributions depend on the existence of the constant term $c$ but not on its value.

Remark 5 Since the long-run relationship in model (13) is given by

$$
y_{t-1}=\frac{\beta}{1-\alpha} z_{t}+\frac{c}{1-\alpha}+a_{t}+\mathbf{M}
$$

it is possible to estimate $\delta=\frac{\beta}{1-\alpha}$ by simply regressing $y_{t-1}$ on $z_{t}$. It is straightforward to show that the resulting OLS estimator, say $\hat{\delta}^{\prime}$, has an asymptotic distribution given by:

$$
\begin{aligned}
& T\left(\hat{\delta}^{\prime}-\delta\right) \\
& \Rightarrow \frac{\frac{1}{1-\rho} \int B_{2}(r) d B_{1}(r)-\left[\frac{1}{1-\rho} B_{1}(1)-\frac{\beta}{1-\alpha} B_{2}(1)\right] \int B_{2}(r) d r-\frac{1}{2} \frac{\beta}{1-\alpha}\left[B_{2}^{2}(1)+\sigma_{2}^{2}\right]}{(1-\alpha)\left[\int B_{2}^{2}(r) d r-\left(\int B_{2}(r) d r\right)^{2}\right]} .
\end{aligned}
$$

The asymptotic distributions given in (16) and (19) are slightly different (note that $\mu$ in (16) is not included in (19)).

\section{Alternative Estimator of the Long-run Co- efficient}

In this section, we introduce an alternative estimator of $\delta$, but for simplicity we deal with $A D L(p, q)$, such as:

$$
\begin{aligned}
y_{t} & =c+A(L) y_{t}+B(L) z_{t}+u_{t}, \\
z_{t} & =z_{t-1}+\varepsilon_{t}, u_{t}=C^{*}(L) u_{t}+v_{t}, \\
t & =1,2, \cdots, T .
\end{aligned}
$$


We have seen that the serial correlation in $u_{t}$ causes the inconsistency of the OLS estimators $\hat{c}, \hat{\alpha}$ and $\hat{\beta}$ in the previous section. To obtain a consistent estimator, we transform the model to eliminate the serial correlation before applying OLS. This can be done by multiplying both sides of (20) by $C^{* *}(L) \equiv\left(1-C^{*}(L)\right)$ and rearranging terms to obtain

$$
\begin{aligned}
y_{t} & =\mu^{*}+A^{*}(L) y_{t}+B^{*}(L) z_{t}+v_{t}, \\
z_{t} & =z_{t-1}+\varepsilon_{t}, \\
t & =1,2, \cdots, T,
\end{aligned}
$$

where

$$
\begin{aligned}
A^{*}(L) & =1-C^{* *}(L)+C^{* *}(L) A(L)=\alpha_{1}^{*} L+\alpha_{2}^{*} L^{2}+\cdots+\alpha_{p^{*}}^{*} L^{p^{*}}, \\
B^{*}(L) & =C^{* *}(L) B(L)=\beta_{0}^{*}+\beta_{1}^{*} L+\cdots+\beta_{q^{*}}^{*} L^{q^{*}}, \\
\mu^{*} & =c C^{* *}(1), p^{*}=p+l, q^{*}=q+l .
\end{aligned}
$$

In this model, the long-run effect of $z$ on $y$ is defined as before, namely

$$
\delta=\frac{B^{*}(1)}{1-A^{*}(1)}
$$

It is straightforward to see that

$$
\frac{B^{*}(1)}{1-A^{*}(1)}=\frac{C^{* *}(1) B(1)}{1-\left[1-C^{* *}(1)+C^{* *}(1) A(1)\right]}=\frac{B(1)}{1-A(1)}=\delta .
$$

We propose an alternative estimator defined by

$$
\check{\delta}=\frac{\check{B}^{*}(1)}{1-\check{A}^{*}(1)}=\frac{\sum_{j=0}^{q+l} \check{\beta}_{j}^{*}}{1-\sum_{i=1}^{p+l} \check{\alpha}_{i}^{*}},
$$

where $\check{\alpha}_{i}^{*}, i=1, \cdots, p+l$, and $\check{\beta}_{j}^{*}, j=0,1, \cdots, q+l$, are the $O L S$ estimators for model (21). As this transformation makes $v_{t}$ i.i.d. $N\left(0, \sigma_{v}^{2}\right)$, it is independent of $y_{t-1}$ and $z_{t}$, so that the $O L S$ estimators $\check{\alpha}_{i}^{*}$ and $\beta_{j}^{*}, i=1,2, \cdots, p+l$, $j=0,1, \cdots, q+l$, are consistent. Therefore, $\check{\delta}$ is also a consistent estimator. We would expect $\delta$ to have better distributional properties than $\hat{\delta}$ in small samples because the $O L S$ estimators, $\hat{\alpha}_{i}$ and $\hat{\beta}_{i}$, are inconsistent.

Using a similar method to that employed in Section 3, we present the following Theorem. 
Theorem 7 For model (21), the asymptotic distribution of the estimator $\check{\delta}$ defined in (23) is given as:

$$
T(\check{\delta}-\delta) \Rightarrow \frac{C(1)}{1-A(1)} \frac{\int B_{2}(r) d B_{1}(r)-B_{1}(1) \int B_{2}(r) d r}{\left(\int B_{2}^{2}(r) d r-\left[\int B_{2}(r) d r\right]^{2}\right)} .
$$

$\mathbf{P}$ roof. Available on request, as an extension of HMM.

\section{Simulation Experiments}

Although we have shown that $\hat{\delta}$ and $\check{\delta}$ are $T$-consistent, and both $T(\check{\delta}-\delta)$ and $T(\hat{\delta}-\delta)$ converge to non-standard distributions, we do not yet know the small sample properties of $\hat{\delta}$ and $\breve{\delta}$.

To investigate the finite sample performance, we conducted some Monte Carlo experiments by using the $\operatorname{ADL}(2,1)$ model, which can be transformed as:

$$
\begin{aligned}
& y_{t}=a_{0}+a_{1} y_{t-1}+a_{2} y_{t-2}+a_{3} z_{t}+a_{4} z_{t-1}+v_{t} \\
& z_{t}=z_{t-1}+\varepsilon_{t}, t=1,2, \cdots, T .
\end{aligned}
$$

In the experiments, we fix the parameters as follows:

$$
a_{0}=c(1-\rho), a_{1}=\alpha+\rho, a_{2}=-\alpha \rho, a_{3}=\beta, a_{4}=-\beta \rho,
$$

and

$$
\sigma_{2}^{2}=1.0, \sigma_{1}^{2}=0.25, c=1, \alpha=0.38, \beta=0.4, \delta=0.645 .
$$

We specify the other parameters as $\rho=0.0,0.5,0.8 ; T=50,100,500$; and calculate $\hat{d}=(\check{\delta}-\delta) / s_{\delta}$ and $\hat{d}=(\hat{\delta}-\delta) / s_{\hat{\delta}} 5000$ times for each parameter combination, where $s_{\delta}$ and $s_{\hat{\delta}}$ represent the estimated standard errors.

Figures 1 through 9 in Appendix are the empirical distributions obtained from the experiments. From these figures we observe that $\delta$ is almost unbiased, but $\hat{\delta}$ is slightly biased in small samples. The bias does not vary greatly as the sample size increases, but increases with $\rho$.

To compare the performance of $\breve{\delta}$ and $\hat{\delta}$, we calculated the sample mean squared errors $(M S E)$ :

$$
\check{e}=\frac{1}{5000} \sum_{i=1}^{5000}\left(\check{\delta}_{i}-\delta\right)^{2}, \hat{e}=\frac{1}{5000} \sum_{i=1}^{5000}\left(\hat{\delta}_{i}-\delta\right)^{2} .
$$


The calculated values of $\check{e}$ and $\hat{e}$ are given in Table 1 .

Table 1. Comparison of $\check{\delta}$ and $\hat{\delta}$ by the $M S E$ criterion

\begin{tabular}{|ccccc|}
\hline$\rho$ & MSE & $\mathrm{T}=50$ & $\mathrm{~T}=100$ & $\mathrm{~T}=500$ \\
0.0 & $\hat{e}$ & 0.003146 & 0.000772 & 0.000003 \\
& $\check{e}$ & 0.003593 & 0.000813 & 0.000003 \\
0.5 & $\hat{e}$ & 0.013747 & 0.003642 & 0.000150 \\
& $\check{e}$ & 0.013722 & 0.003041 & 0.000115 \\
0.8 & $\hat{e}$ & 0.101997 & 0.027711 & 0.001292 \\
& $\check{e}$ & 0.116559 & 0.018894 & 0.000689 \\
\hline
\end{tabular}

It can be seen that the difference between $\check{e}$ and $\hat{e}$ becomes large as $\rho$ increases. Moreover, the values of $\hat{e}$ are generally larger than those of $\check{e}$ if $\rho \neq 0$, the difference becoming small as the sample size increases. Judging by the $M S E$ criterion, we can say that $\delta$ is better than $\delta$. HMM also compared the two estimators in the simplest case, such as the $A D L(1,0)$ model, and obtained similar results.

\section{Concluding Remarks}

In this paper, we developed an asymptotic theory for the estimators in a general $A D L(p, q)$ model with integrated regressors and serially correlated disturbances, found that the $O L S$ estimators $\hat{c}, \hat{\alpha}$ and $\hat{\beta}$ for the regression coefficients are $\sqrt{T}-$ inconsistent but have asymptotic normal distributions, and that the $O L S$-based estimator $\hat{\delta}$ for the long-run coefficient is $T$-consistent, i.e., superconsistent, but with a nonstandard asymptotic distribution. Therefore, standard statistical inference which relies on asymptotic normality for the regression coefficients and the long-run coefficient are misleading. Furthermore, we proposed an alternative estimator $\delta$ for the long-run coefficient obtained by transforming the original model to eliminate the serial correlation in the disturbances, and examined the asymptotic properties of the proposed estimator. Monte Carlo experiments show that the proposed estimator $\check{\delta}$ is $M S E$ - superior to the $O L S$-based estimator. 


\section{References}

[1] He, Z, Maekawa. K., and M. McAleer, (1998), Asymptotic Properties of the Estimated Long-Run MPC in a Dynamic Model with an Integrated Regressor, Working Paper, Hiroshima University.

[2] Maekawa, K., T. Yamamoto, Y. Takeuchi, and M. Hatanaka, (1996), Estimation in Dynamic Regression with an Integrated Process, Journal of Statistical Planning and Inference, 49, 279-303.

[3] Park, J.Y. and P.C.B. Phillips, (1989) Statistical Inference in Regressions with Integrated Process: Part 2, Econometric Theory, 5, 95-131.

[4] Phillips, P.C.B., (1987), Time Series Regression with a Unit Root, Econometrica, 55, 277-301. 
Figure 1. $(T=50, \rho=0.0)$

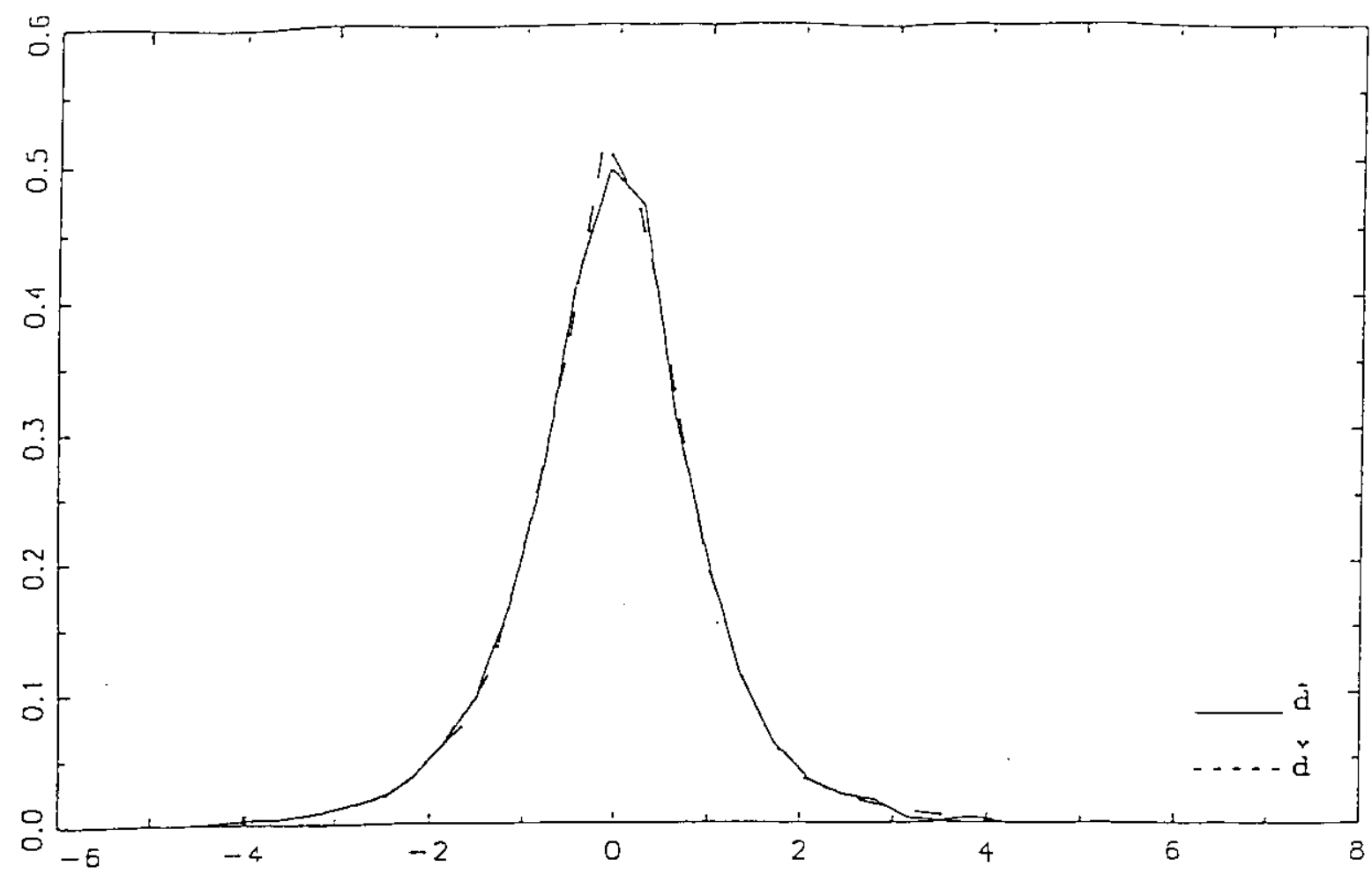

Figure 2. $(T=50, \rho=0.5)$

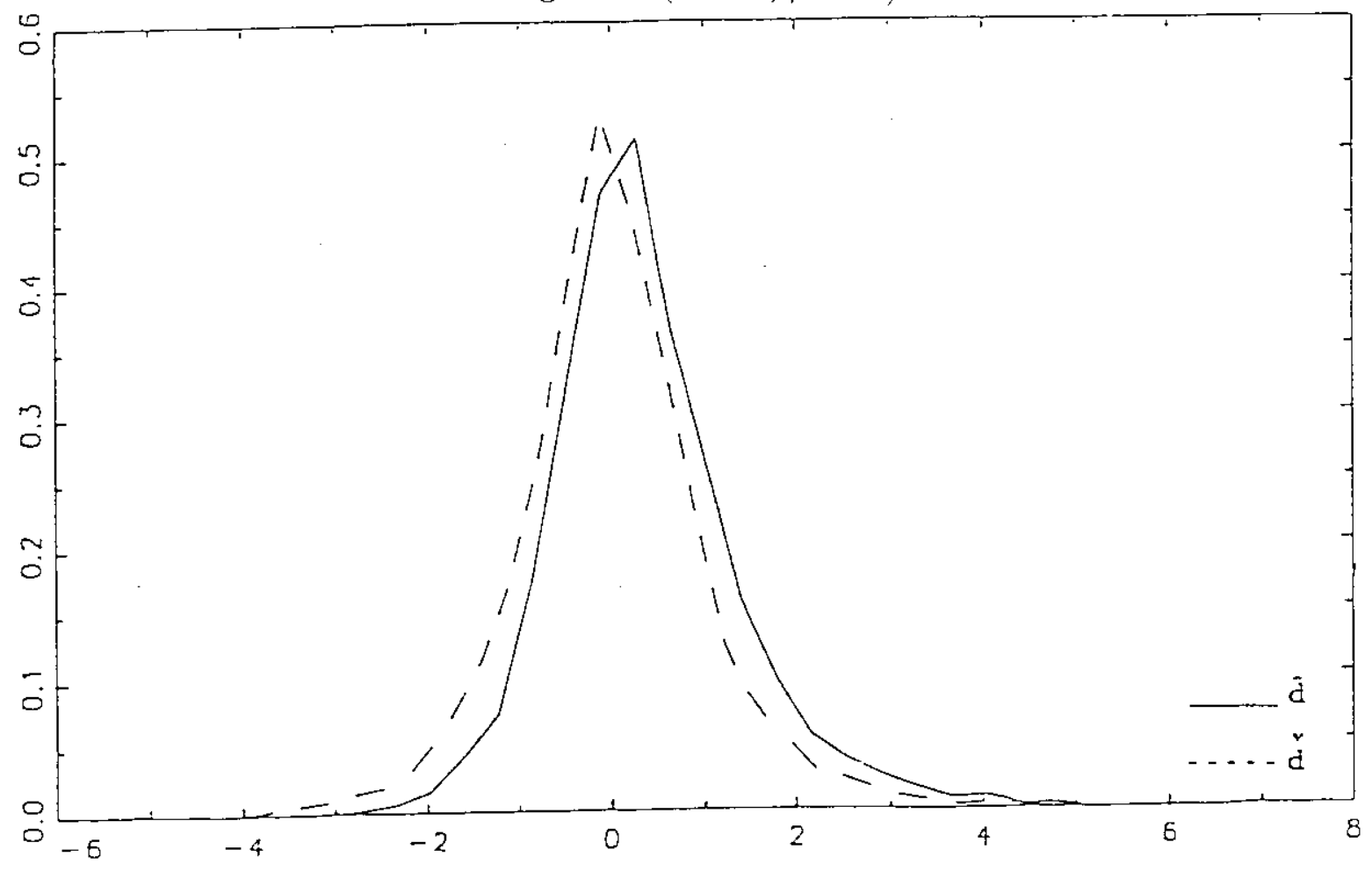


Figure 3. $(T=50, \rho=0.8)$
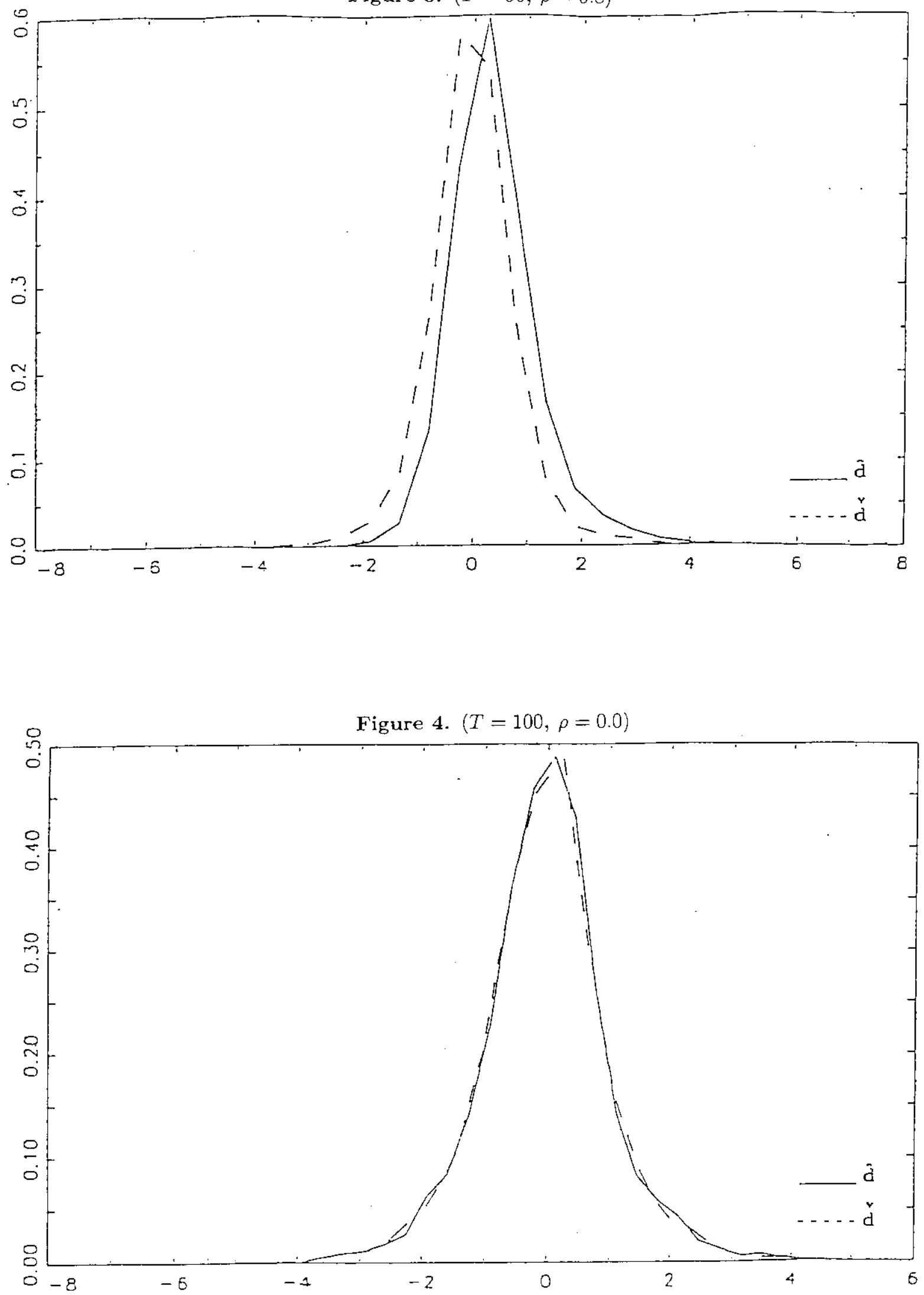
Figure 5. $(T=100, \rho=0.5)$
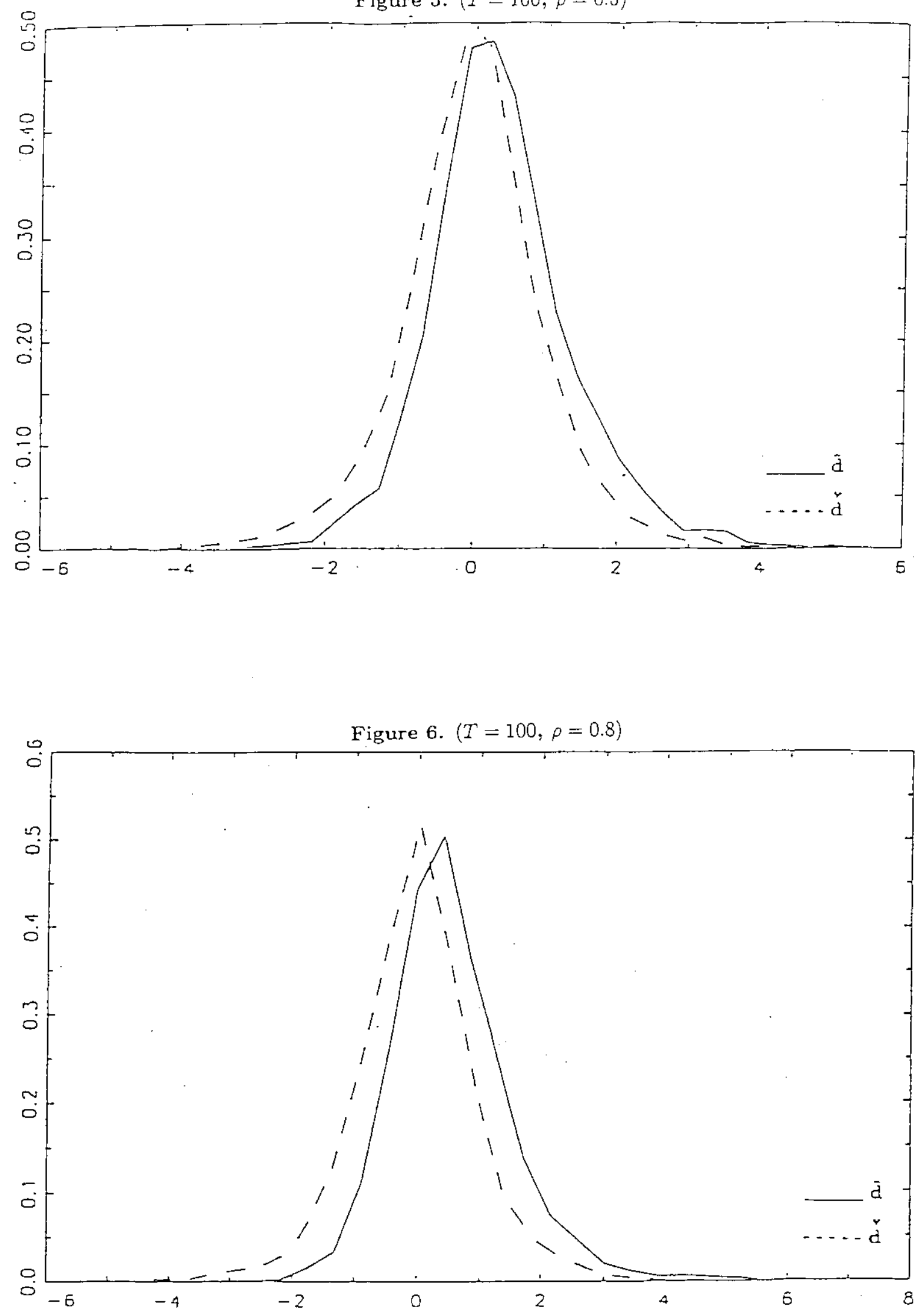
Figure 7. $(T=500, \rho=0.0)$

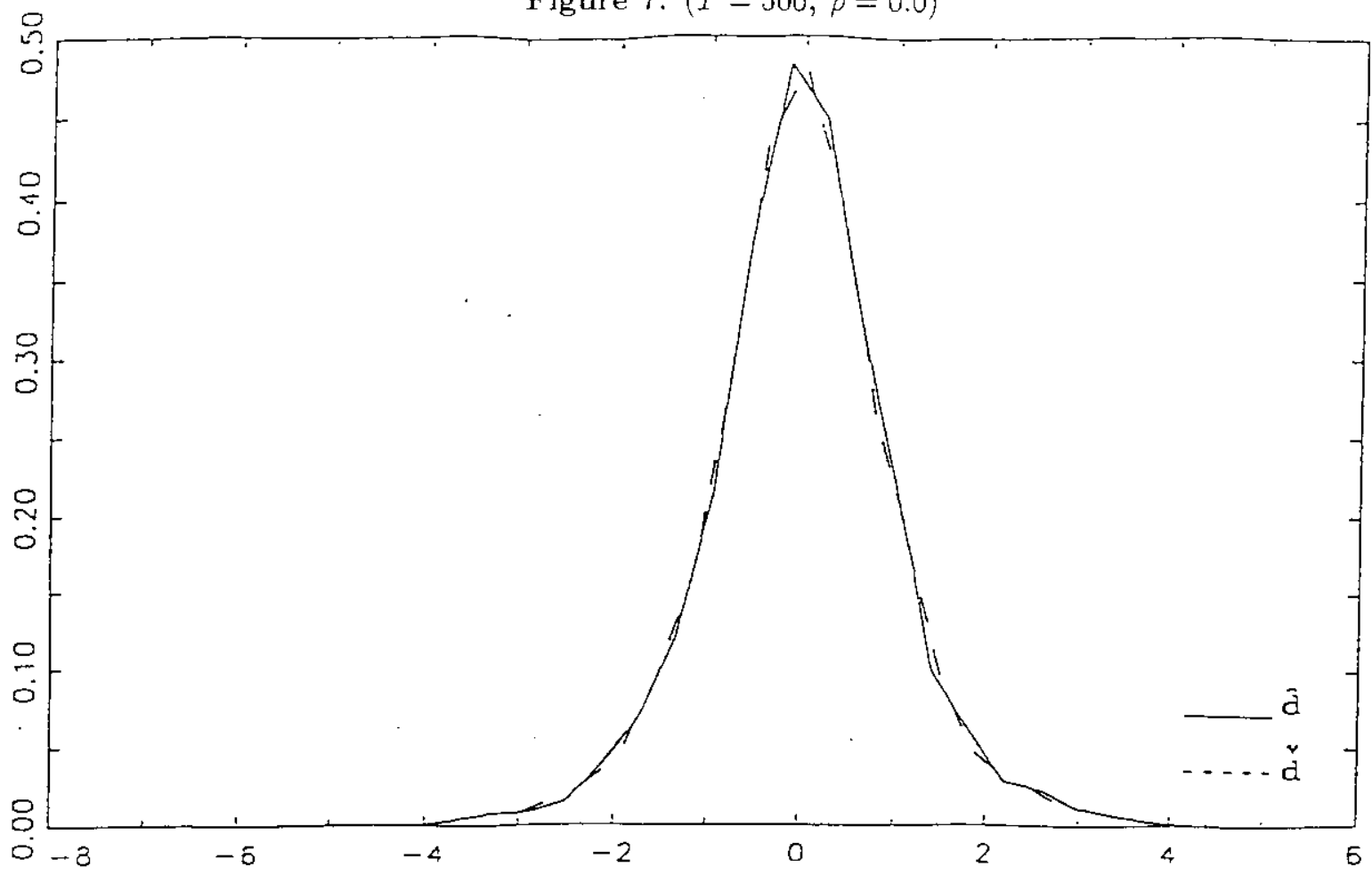

Figure 8. $(T=500, \rho=0.5)$

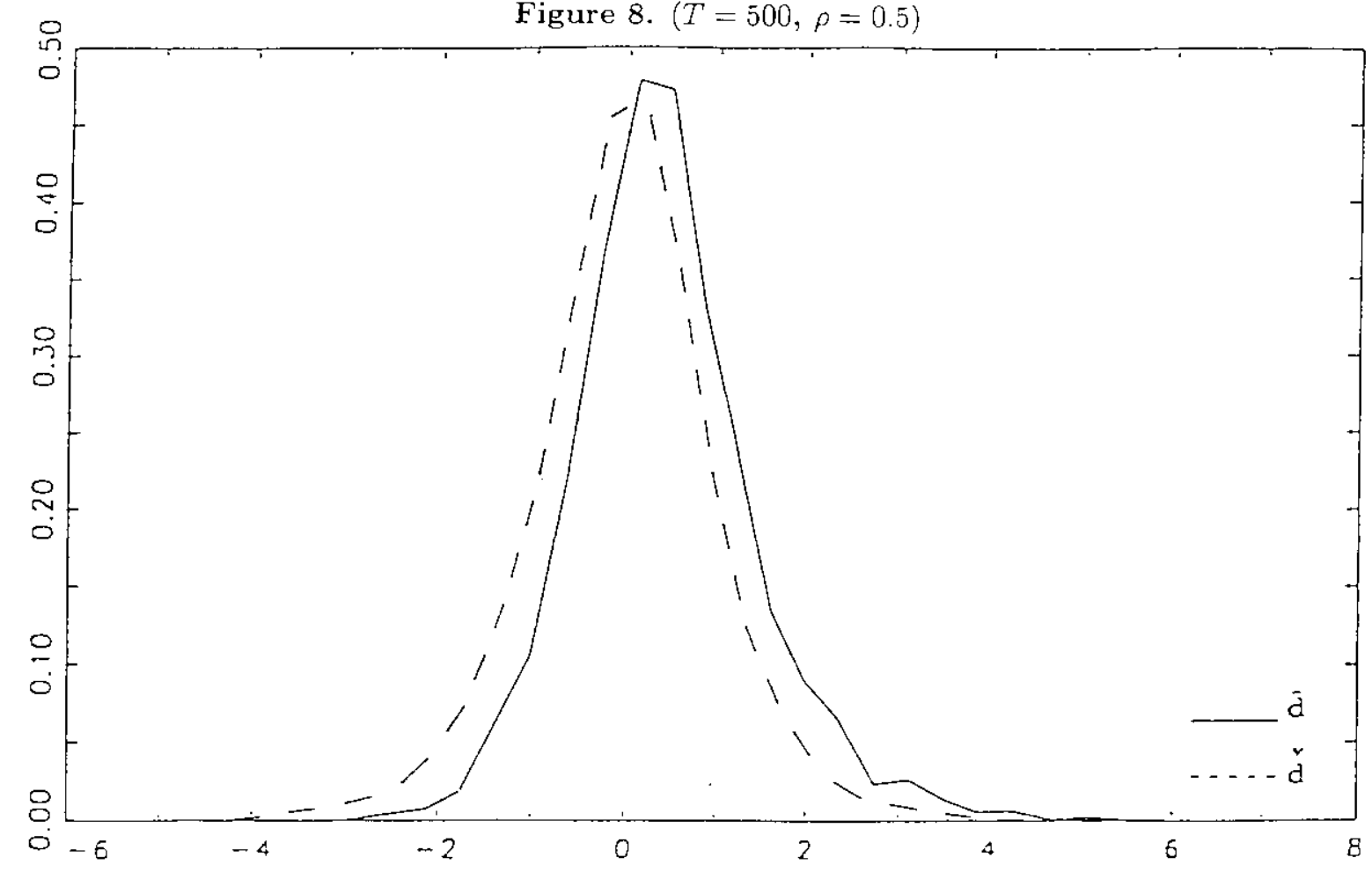


Figure 9. $(T=500, \rho=0.8)$

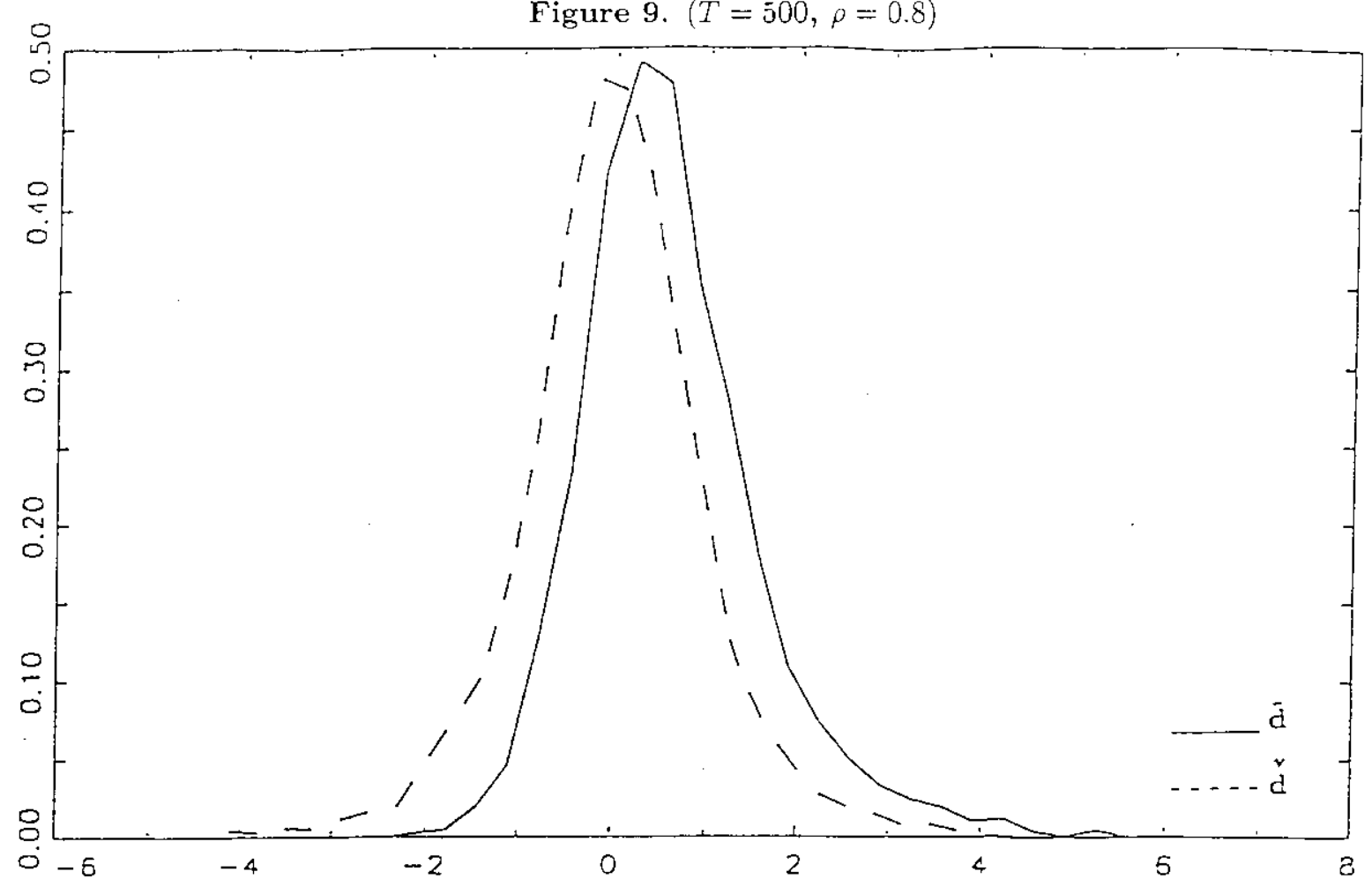

